\title{
Progression to the Persistent Form in Asymptomatic Paroxysmal Atrial Fibrillation
}

\author{
Keitaro Senoo, MD; Shinya Suzuki, MD; Takayuki Otsuka, MD; Koichi Sagara, MD; \\ Shunsuke Matsuno, MD; Hiroto Kano, MD; Tokuhisa Uejima, MD; \\ Yuji Oikawa, MD; Junji Yajima, MD; Kazuyuki Nagashima, MD; Hajime Kirigaya, MD; \\ Hitoshi Sawada, MD; Tadanori Aizawa, MD; Takeshi Yamashita, MD
}

\begin{abstract}
Background: This study investigated the progression of paroxysmal atrial fibrillation (AF) to the persistent form in Japanese asymptomatic AF patients.

Methods and Results: Data were derived from a single hospital-based cohort in the Shinken Database 2004-2012 $(\mathrm{n}=19,994)$, in which 1,176 patients were diagnosed as having paroxysmal AF. AF progression occurred in 115 patients $(6.0 \% /$ year) during the mean follow-up period (1,213 \pm 905 days). Although patients who were asymptomatic at the initial visit $(n=468)$ had a low-risk profile compared with symptomatic patients, they had greater AF progression at follow-up (unadjusted hazard ratio, 1.611; 95\% Cl: 1.087-2.389; $\mathrm{P}=0.018$ ). Absence of symptoms, male sex, and cardiomyopathy were independent predictors for AF progression in the multivariate model. It was noted that asymptomatic patients were less likely to undergo pulmonary vein isolation (PVI). In addition, the interaction term between asymptomatic AF and absence of PVI could be another independent predictor for AF progression. Prognosis was similar between asymptomatic and symptomatic patients with AF.
\end{abstract}

Conclusions: Irrespective of low-risk profile, patients with asymptomatic paroxysmal AF had greater progression of $A F$ compared with symptomatic patients. This paradoxical result appeared to be the result of less intensive clinical management, including invasive rhythm control. (Circ $J$ 2014; 78: 1121-1126)

Key Words: Asymptomatic atrial fibrillation; Atrial fibrillation; Epidemiology; Progression

A trial fibrillation $(\mathrm{AF})$ is the most common clinically significant arrhythmia in clinical practice. ${ }^{1} \mathrm{AF}$ is associated with increased morbidity and mortality that primarily occur as a result of 2 complications: stroke and heart failure. ${ }^{2}$

\section{Editorial p 1058}

In clinical practice, 1 should distinguish between paroxysmal $\mathrm{AF}$ (episodes of arrhythmia that terminate spontaneously) and persistent $\mathrm{AF}$ (episodes that are sustained for $>7$ days and are not self-terminating). ${ }^{3}$ Paroxysmal AF often progresses into persistent AF. ${ }^{4,5}$ Independent predictors of AF progression include older age and heart disease, which cause atrial structural remodeling. ${ }^{6}$ Although AF causes symptoms in most patients, it can be diagnosed when patients are asymptomatic. ${ }^{7}$ In our previous report, patients with symptomatic $\mathrm{AF}$ were older, had a frequent incidence of heart failure, and had large left atrial dimension, suggesting progression of atrial structural remodeling. ${ }^{8}$ Further- more, it is generally believed that frequent AF symptoms lead to an increased incidence of AF episodes, and could thus predict AF progression. An epidemiological study, however, recently reported that asymptomatic $\mathrm{AF}$ is associated with future AF progression compared with symptomatic AF. ${ }^{9}$

Current evidence is scarce, and the impact of asymptomatic AF on AF progression and its clinical implication in the Japanese clinical setting remain unclear. The present study was designed to investigate AF progression and clinical outcome in asymptomatic Japanese patients with paroxysmal AF compared with symptomatic patients in a large, single hospital-based cohort study from the Shinken Database.

\section{Methods}

\section{Subjects}

The Shinken Database consists of all new patients visiting the Cardiovascular Institute Hospital in Tokyo, Japan ("Shinken" is an abbreviated name in Japanese for the name of the hospital).

Received October 11, 2013; revised manuscript received December 25, 2013; accepted January 7, 2014; released online February 20,2014 Time for primary review: 24 days

Cardiovascular Institute, Tokyo, Japan

Mailing address: Keitaro Senoo, MD, Department of Cardiology, Cardiovascular Institute, 3-2-19 Nishiazabu, Minato-Ku, Tokyo 106-0031, Japan. E-mail: swcqg251@yahoo.co.jp

ISSN-1346-9843 doi:10.1253/circj.CJ-13-1272

All rights are reserved to the Japanese Circulation Society. For permissions, please e-mail: cj@j-circ.or.jp 


\begin{tabular}{|c|c|c|c|c|}
\hline \multirow[b]{2}{*}{ Characteristics } & \multicolumn{2}{|c|}{ Paroxysmal AF } & \multirow[b]{2}{*}{ P-value } & \multirow[b]{2}{*}{$\begin{array}{c}\text { Overall } \\
(n=1,176)\end{array}$} \\
\hline & $\begin{array}{c}\text { Asymptomatic } \\
(n=468)\end{array}$ & $\begin{array}{c}\text { Symptomatic } \\
(n=708)\end{array}$ & & \\
\hline Age (years) & $61.2 \pm 12.6$ & $61.5 \pm 13.5$ & 0.709 & $61.4 \pm 13.1$ \\
\hline Male & $373(79.3)$ & $502(70.9)$ & 0.001 & $875(74.4)$ \\
\hline BMI & $23.8 \pm 3.2$ & $23.4 \pm 3.3$ & 0.095 & $23.6 \pm 3.2$ \\
\hline Hypertension & $179(38.2)$ & $318(44.9)$ & 0.026 & $497(42.3)$ \\
\hline Dyslipidemia & $120(25.6)$ & $175(24.7)$ & 0.732 & $295(25.1)$ \\
\hline Diabetes mellitus & $48(10.3)$ & $85(12.0)$ & 0.397 & $133(11.3)$ \\
\hline \multicolumn{5}{|l|}{ Cardiovascular disease } \\
\hline Structural heart disease & 91 (19.4) & $130(18.4)$ & 0.648 & $221(18.8)$ \\
\hline Ischemic heart disease & $36(7.7)$ & $48(6.8)$ & 0.565 & $84(7.1)$ \\
\hline Valvular disease & $33(7.1)$ & 49 (6.9) & 1.0 & $82(7.0)$ \\
\hline Cardiomyopathy & $31(6.6)$ & $45(6.4)$ & 0.904 & $76(6.5)$ \\
\hline History of congestive heart failure & $42(9.0)$ & 77 (10.9) & 0.324 & $119(10.1)$ \\
\hline History of TIA/stroke & $14(3.0)$ & $30(4.2)$ & 0.346 & $44(3.7)$ \\
\hline CHADS $_{2}$ score & $0.8 \pm 1.0$ & $0.9 \pm 1.0$ & 0.015 & $0.9 \pm 1.0$ \\
\hline 0 & $232(49.6)$ & $296(41.8)$ & 0.032 & $528(44.9)$ \\
\hline 1 & $138(29.5)$ & 240 (33.9) & & $378(32.1)$ \\
\hline$\geq 2$ & $98(20.9)$ & $172(24.3)$ & & $270(23.0)$ \\
\hline $\mathrm{CHA}_{2} \mathrm{DS}_{2}$-VASc score & $1.4 \pm 1.5$ & $1.7 \pm 1.5$ & 0.05 & $1.6 \pm 1.5$ \\
\hline 0 & $160(34.2)$ & $188(26.6)$ & 0.012 & $348(29.6)$ \\
\hline 1 & $121(25.9)$ & $187(26.4)$ & & $308(26.2)$ \\
\hline$\geq 2$ & $187(40.0)$ & $333(47.0)$ & & $520(44.2)$ \\
\hline \multicolumn{5}{|l|}{ Medication at discharge } \\
\hline Antiplatelet agents & $177(40.9)$ & 291 (39.2) & 0.579 & 468 (39.8) \\
\hline Anticoagulants & $158(33.8)$ & $300(42.4)$ & 0.006 & $458(39.0)$ \\
\hline Warfarin & $150(32.1)$ & $234(33.1)$ & 0.751 & $384(32.7)$ \\
\hline Dabigatran & $8(1.7)$ & $66(9.3)$ & $<0.001$ & $74(6.3)$ \\
\hline$\beta$-blockers & $130(27.8)$ & $229(32.3)$ & 0.106 & $359(30.5)$ \\
\hline Calcium channel blocker & $58(12.4)$ & $90(12.7)$ & 0.929 & $148(12.6)$ \\
\hline Digitalis glycosides & $25(5.3)$ & $42(5.9)$ & 0.702 & $67(5.7)$ \\
\hline Diuretics & $42(9.0)$ & $79(11.2)$ & 0.241 & $121(10.3)$ \\
\hline RAS inhibitors & $91(19.4)$ & $161(22.7)$ & 0.191 & $252(21.4)$ \\
\hline Class la/lc & $205(43.8)$ & $317(44.8)$ & 0.764 & $522(44.4)$ \\
\hline \multicolumn{5}{|l|}{ Echocardiogram parameters ${ }^{\dagger}$} \\
\hline LVEF (\%) & $64.9 \pm 9.6$ & $65 \pm 10.8$ & 0.830 & $65 \pm 10.3$ \\
\hline Left atrial dimension (mm) & $37.8 \pm 6.6$ & $37.6 \pm 6.8$ & 0.531 & $37.7 \pm 6.7$ \\
\hline \multicolumn{5}{|l|}{ Holter recording parameters ${ }^{\ddagger}$} \\
\hline Paroxysmal AF (\%) & $57(33.1)$ & $63(30.4)$ & 0.581 & $120(31.7)$ \\
\hline All-day AF during recording & $7(4.1)$ & $7(3.4)$ & 0.788 & $14(3.7)$ \\
\hline
\end{tabular}

Data given as mean \pm SD or $\mathrm{n}(\%)$. ${ }^{\dagger}$ Obtained for 412,617 and 1,029 patients in asymptomatic, symptomatic and overall groups, respectively. $¥$ Obtained for 172,207 and 379 patients in asymptomatic, symptomatic and overall groups, respectively. AF, atrial fibrillation; $\mathrm{BMI}$, body mass index; $\mathrm{CHADS}_{2}$ score, congestive heart failure, hypertension, age $>75$, diabetes mellitus, stroke or TIA; $\mathrm{CHA}_{2} \mathrm{DS}_{2}$-VASc score, congestive heart failure, hypertension, age $>75$, diabetes mellitus, stroke or TIA, vascular disease, age 65-74 years and sex category (female gender); LVEF, left ventricular ejection fraction; RAS, renin-angiotensin system; TIA, transient ischemic attack.

It excludes patients with active cancer and foreign travelers. The principal aim of this hospital-based database is surveillance of the prevalence and prognosis of cardiovascular disease in the urban areas of Japan. ${ }^{10-14}$ The registry began in June 2004, and thereafter, patients have continually been registered to the database every year. Health status, incidence of cardiovascular events, and mortality are updated in the database by linking to the medical records of the hospital and by mailings sent to patients approximately once or twice per year.

The data in the present study were derived from this database between 2004 and 2012 (Shinken Database 2004-2012), con- sisting of 19,994 new visiting patients. In the database, 2,333 patients were diagnosed with AF, of whom 1,176 patients had paroxysmal $\mathrm{AF}$ and had a mean follow-up period of 1,213 \pm 905 days. These patients comprised the present study group.

\section{Data Collection}

After electrocardiogram (ECG) and chest X-ray, cardiovascular status was evaluated for each patient using echocardiography, exercise test, 24-h Holter recordings, and blood laboratory data from the initial visit, as determined by the attending physicians. From the database, the following information was collected: 


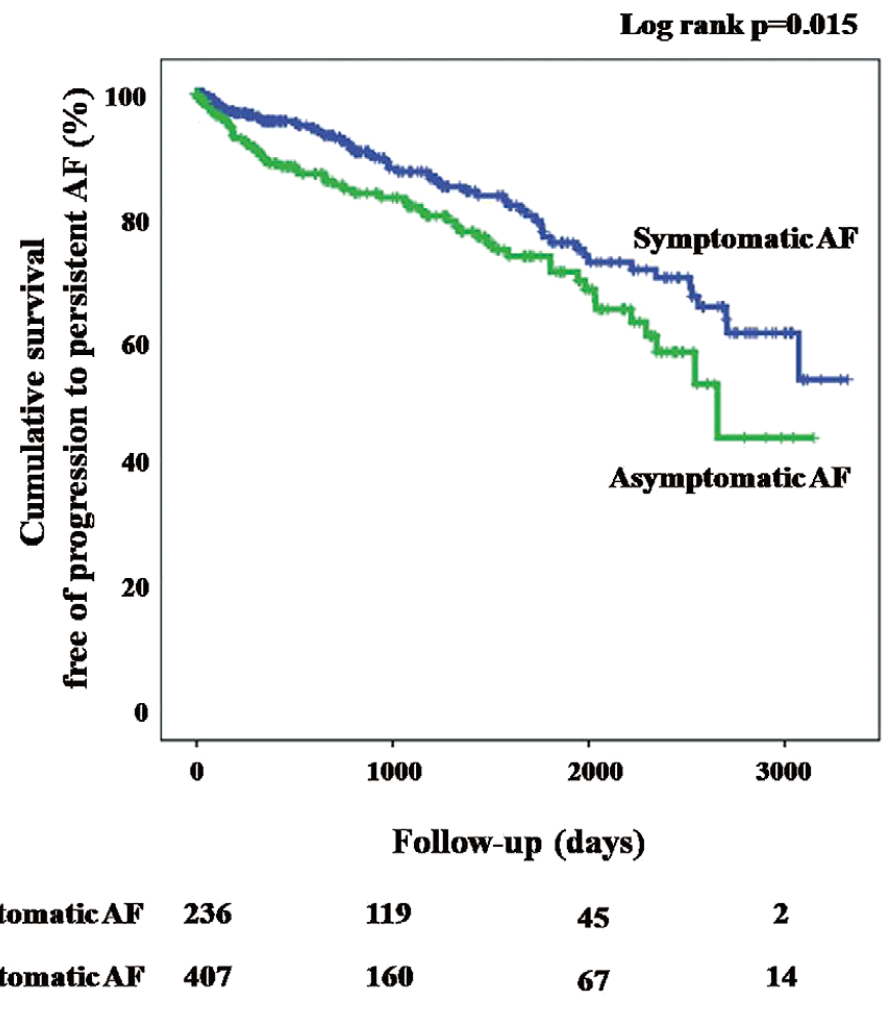

Figure. Kaplan-Meier curve of progressionfree survival in patients with asymptomatic and symptomatic paroxysmal atrial fibrillation $(\mathrm{AF})$.

(1) patient data, including sex, age, height, and weight; (2) cardiovascular risk factors, including hypertension (use of antihypertensive agents, systolic blood pressure $\geq 140 \mathrm{mmHg}$, or diastolic blood pressure $90 \mathrm{mmHg}$ on admission) and diabetes mellitus (use of oral hypoglycemic agents or insulin, or glycosylated hemoglobin $\geq 6.5 \%$ ); (3) cardiovascular disease, including structural heart disease, congestive heart failure, or a history of a disabling cerebral infarction or transient ischemic attack (TIA); and (4) use of medication. Body mass index (BMI) was calculated as weight in kilograms divided by the square of height in meters.

\section{Definitions}

In the present study, paroxysmal $\mathrm{AF}$ at the initial visit was defined as (1) sinus rhythm on ECG and previous diagnosis of paroxysmal AF by referring physicians; (2) symptomatic AF on ECG at the initial visit and duration of AF estimated as $<7$ days according to symptoms or ECG recordings; and (3) asymptomatic AF on ECG at the initial visit and no AF 1 week prior. Patients whose AF was estimated to continue for $>7$ days after the initial visit, were considered to have persistent AF originally and were excluded from the analysis.

Asymptomatic AF was defined as AF documented on 12lead ECG during a visit, in the absence of any new symptoms such as palpitations, tachycardia, fatigue, malaise, shortness of breath on exertion, dyspnea, chest pain, syncope, or presyncope related to $\mathrm{AF}$ and other illnesses.

During the follow-up period, the onset of persistent AF was defined as the first time in which all ECG indicated AF after $\geq 3$ consecutive ECG at an interval $\geq 1$ week after the initial examination. When an ECG could not be obtained thrice during the period, the physicians made a clinical judgment regarding the onset time of AF progression. When electrical cardioversion was performed after $>7$ days continuing from $\mathrm{AF}$ onset, it was also considered as AF progression.

We calculated the $\mathrm{CHADS}_{2}$ score $^{15}$ (congestive heart failure, hypertension, age [ $\geq 75$ years], diabetes mellitus, and history of stroke or TIA; 2 points). Also, the $\mathrm{CHA}_{2} \mathrm{DS}_{2}-\mathrm{VASc}$ score was determined, adding vascular disease (previous myocardial infarction, complex aortic plaque, and peripheral artery disease [PAD]), age 65-74, and female sex to the factors of the original CHADS 2 score. ${ }^{16}$

\section{Statistical Analysis}

For baseline characteristics, continuous variables and categorical variables are expressed as mean $\pm \mathrm{SD}$ and as absolute numbers (percentages), respectively. Chi-squared test and unpaired Student's t-test were used for the comparison of categories and continuous variables between 2 groups, respectively. Cumulative progression-free survival (ie, without progression to persistent AF) was estimated using the Kaplan-Meier method. Univariate and multivariate Cox regression analyses were performed to identify predictors of AF progression. In the multivariate model, cofactors that were significantly associated with AF progression in the univariate models were analyzed using the stepwise method (model 1). The multivariate model 2 was specially developed to determine whether the interaction between asymptomatic AF and the effect of treatment intervention on AF progression was significant. The incidence of adverse events (eg, AF progression, ischemic stroke, cardiovascular death, and all-cause death) was calculated by the person-year method. All statistical analysis was done using SPSS version 19.0 (SPSS, Chicago, IL, USA) for Windows (Microsoft Corp, Redmond, WA, USA). Statistical significance was set at $\mathrm{P}<0.05$. 


\begin{tabular}{|llll|}
\hline Table 2. Predictors of Progression to Persistent AF & & & \\
& HR & 95\% Cl & P-value \\
Univariate model & & & \\
Age & 1.014 & $0.998-1.031$ & 0.082 \\
Sex (male) & 2.063 & $1.217-3.500$ & 0.007 \\
Asymptomatic AF & 1.611 & $1.087-2.389$ & 0.018 \\
Hypertension & 0.963 & $0.661-1.402$ & 0.844 \\
Dyslipidemia & 1.117 & $0.751-1.662$ & 0.585 \\
Diabetes mellitus & 1.472 & $0.889-2.439$ & 0.133 \\
Structural heart disease & 1.329 & $0.893-1.979$ & 0.161 \\
Ischemic heart disease & 1.038 & $0.542-1.988$ & 0.911 \\
Valvular disease & 0.95 & $0.532-1.697$ & 0.863 \\
Cardiomyopathy & 2.125 & $1.265-3.568$ & 0.004 \\
History of stroke or TIA & 1.901 & $0.833-4.336$ & 0.127 \\
History of heart failure & 1.304 & $0.812-2.095$ & 0.273 \\
Absence of PVI & 1.703 & $0.983-2.948$ & 0.057 \\
Multivariate model 1 & & & \\
Asymptomatic AF & 1.461 & $1.007-2.119$ & 0.046 \\
Cardiomyopathy & 1.882 & $1.117-3.173$ & 0.018 \\
Sex (male) & 1.872 & $1.009-3.190$ & 0.021 \\
Multivariate model 2 & & & \\
Asymptomatic AFxabsence of PVI ${ }^{\dagger}$ & 1.490 & $1.020-2.175$ & 0.039 \\
Cardiomyopathy & 1.849 & $1.095-3.120$ & 0.021 \\
Sex (male) & 1.904 & $1.119-3.241$ & 0.018 \\
\hline
\end{tabular}

†Interaction term. $\mathrm{AF}$, atrial fibrillation; $\mathrm{Cl}$, confidence interval; $\mathrm{HR}$, hazard ratio; $\mathrm{PVI}$, pulmonary vein isolation. Other abbreviations as in Table 1.

\section{Ethics}

The ethics committee of the Cardiovascular Institute granted ethics permission for this study, and all patients provided written informed consent.

\section{Results}

\section{Subject Characteristics}

The baseline characteristics of the 1,176 patients with paroxysmal AF are listed in Table 1. The mean age of all patients was $61.4 \pm 13.1$ years. Mean BMI was 23.6 \pm 3.2 . A total of 468 patients $(38.9 \%)$ were asymptomatic. Among asymptomatic and symptomatic patients with $\mathrm{AF}$, there were similar prevalences of dyslipidemia, diabetes mellitus, and cardiovascular diseases (eg, structural heart disease, congestive heart failure, and TIA/ stroke). Asymptomatic patients with AF were more likely to be male and had a lower prevalence of hypertension, resulting in a greater prevalence of a $\mathrm{CHADS}_{2}$ score of 0 and $\mathrm{CHA}_{2} \mathrm{DS}_{2}-\mathrm{VASc}$ score of 0 (both $\mathrm{P}<0.05$ ). At baseline, fewer asymptomatic patients received anticoagulation than did symptomatic patients. Otherwise, there were no substantial differences in medication use (eg, antiplatelet and anti-arrhythmic drugs [class Ia/Ic]).

In addition, echocardiogram parameters and Holter recordings were compared between asymptomatic and symptomatic patients, although these parameters were obtained in a limited number of patients. Echocardiogram parameters, including left ventricular ejection fraction and left atrial dimension, were similar between asymptomatic and symptomatic AF patients. Moreover, short AF episodes and all-day AF during Holter recording were similarly documented between asymptomatic and symptomatic $\mathrm{AF}$ patients (detection rates of short $\mathrm{AF}$ episodes, $33.1 \%$ vs. $30.4 \%$; $\mathrm{P}=0.581$; all-day $\mathrm{AF}$ during Holter recording, $4.1 \%$ vs. $3.4 \%$; $\mathrm{P}=0.788$, respectively).

\section{Follow-up}

During the follow-up period of 1,213 \pm 905 days, AF progression occurred in 115 patients (6.0\%/year). This was observed more in asymptomatic than in symptomatic patients ( $8.6 \%$ vs. $4.7 \%$ ) year; log rank test, $\mathrm{P}=0.015$; Figure). In the unadjusted model with Cox regression analysis, the hazard ratio (HR) of asymptomatic $\mathrm{AF}$ for $\mathrm{AF}$ progression was 1.611 (95\% confidence interval $[C I]$ : $1.087-2.389 ; \mathrm{P}=0.018)$. In the multivariate model, which used all factors that were significantly associated with AF progression in univariate models (model 1), the independent predictors for AF progression included asymptomatic AF, male sex, and cardiomyopathy (Table 2). Moreover, multivariate model 2 was specially developed to determine whether the interaction between asymptomatic $\mathrm{AF}$ and the effect of treatment intervention on AF progression was significant. For this purpose, we used these models with the interaction term for asymptomatic $\mathrm{AF}$ in model 2, but the absence of treatment intervention (pulmonary vein isolation; PVI) was not significantly associated with AF progression ( $\mathrm{P}=0.057)$, Accordingly, in model 2, the interaction term between the absence of PVI and asymptomatic $\mathrm{AF}$ was identified as an independent predictor for AF progression (Table 2).

The clinical outcome rates during follow-up are given in Table 3 as the crude number of events per 100 patient-years. Of the patients who underwent PVI during the follow-up period, the prevalence was lower in patients who were asymptomatic compared to those who were symptomatic $(69,14.8 \%$ vs. 162 , $22.8 \%$, respectively; $\mathrm{P}=0.001$ ), but there was no significant difference in other clinical outcomes (eg, ischemic stroke, cardiovascular death, and all-cause mortality) between asymptomatic and symptomatic patients with AF. 
Table 3. Clinical Outcome

\begin{tabular}{lcccc} 
& $\begin{array}{c}\text { Asymptomatic AF } \\
(\mathbf{n}=\mathbf{4 6 8 )}\end{array}$ & $\begin{array}{c}\text { Symptomatic AF } \\
(\mathbf{n}=\mathbf{7 0 8})\end{array}$ & P-value & $\begin{array}{c}\text { Overall } \\
(\mathbf{n = 1 , 1 7 6 )}\end{array}$ \\
AF progression & $55(8.6)$ & $60(4.7)$ & 0.001 & $115(6.0)$ \\
Ischemic stroke & $9(0.6)$ & $25(1.1)$ & 0.113 & $34(0.9)$ \\
CV death & $4(0.4)$ & $8(0.5)$ & 0.772 & $12(0.4)$ \\
All-cause death & $10(0.9)$ & $18(1.0)$ & 0.701 & $28(1.0)$ \\
\hline
\end{tabular}

Data given as no. events (crude rate for 100 patient-years). CV, cardiovascular. Other abbreviations as in Table 2.

\section{Discussion}

In the present study involving patients from a single cardiovascular hospital, patients with asymptomatic paroxysmal AF had greater AF progression into the persistent form compared to those with symptomatic AF, irrespective of the patients' lowrisk profiles. When we considered the effect of treatment intervention during follow-up, the interaction term between the absence of PVI and asymptomatic AF was independently associated with AF progression. Furthermore, asymptomatic AF patients had similar mortality rates and incidence rates of ischemic stroke compared to symptomatic ones.

It is generally considered that asymptomatic patients with $\mathrm{AF}$ have few cardiovascular comorbidities compared with symptomatic patients. ${ }^{17}$ It is natural to consider that asymptomatic paroxysmal $\mathrm{AF}$ would progress into the persistent form less frequently and would have a better prognosis than symptomatic AF. In the present study, asymptomatic AF patients had lower $\mathrm{CHADS}_{2}$ and $\mathrm{CHA}_{2} \mathrm{DS}_{2}-\mathrm{VASc}$ scores compared with symptomatic ones, demonstrating the low-risk profile. Paradoxically, however, asymptomatic paroxysmal AF had a greater association with AF progression, and this association remained independent even after adjustment for various clinical patient characteristics. A similar paradoxical association has been reported previously. ${ }^{7}$ In a population-based study with first-detected AF, $\mathrm{AF}$ progression was observed in more patients with asymptomatic paroxysmal AF $(41 / 79 ; 51.9 \%)$ compared to those with symptomatic $\mathrm{AF}(280 / 811 ; 34.5 \%)$, with a significant HR of 1.6 (95\% CI: 1.1-2.2) in the multivariate model. The present results extend these results to Japanese AF patients in a single cardiovascular hospital. The annual incidence rate of AF progression (asymptomatic AF, $8.6 \%$ vs. symptomatic AF, $4.7 \%$, respectively) and the HR of asymptomatic AF in the multivariate model (1.461) in the present patients are similar to those in the previous study. ${ }^{7}$

It is difficult to determine the reason as to why asymptomatic AF patients had a greater association with AF progression irrespective of low-risk profile. From a pathophysiological standpoint, left atrial dimension and left ventricular ejection fraction were similar between the present asymptomatic and symptomatic patients, suggesting that the progression of cardiac remodeling was similar. From a clinical standpoint, although the use of anti-arrhythmic agents (class Ia/Ic) was similar between asymptomatic and symptomatic patients $(43.8 \%$ vs. $44.8 \%$, respectively), the prevalence of PVI was lower among asymptomatic patients than among symptomatic ones (14.8\% vs. $22.8 \%$; $\mathrm{P}=0.001$, respectively). Moreover, in the multivariate model, absence of PVI had a significant interaction with asymptomatic $\mathrm{AF}$, and this interaction term had an independent association with AF progression. In this regard, it is assumed that the effect of asymptomatic AF on AF progression in the present study might be partly explained by a less-invasive intervention for rhythm control.
In the present study, the incidence rates of mortality or the development of ischemic stroke in asymptomatic AF patients were similar to those in symptomatic AF patients, irrespective of the low-risk profile. Consequently, the prognosis for AF appeared to be unrelated to the presence of symptoms. These results are consistent with the results of the Atrial Fibrillation Follow-up Investigation of Rhythm Management (AFFIRM) study ${ }^{18}$ and other trials that support the concept that rate control is an acceptable strategy in the management of AF. Whether active invasive rhythm control, including catheter ablation, is beneficial for patients with asymptomatic paroxysmal AF remains to be investigated.

As with other studies on asymptomatic AF, we found that asymptomatic AF patients were more frequently male. ${ }^{6,8}$ This finding may simply reflect the greater risk for AF progression in male patients. A diagnosis of cardiomyopathy may reflect a variety of etiologies, including hypertension, which is not independently associated with a higher probability of progression. Cardiomyopathy will result in increased left atrial pressure and wall stress, which may be important stimuli for chronic atrial structural remodeling, enabling the maintenance of AF. ${ }^{19}$

\section{Study Limitations}

The present study has several limitations. Because this was a retrospective study, the definition of asymptomatic and paroxysmal AF was not completely accurate. This was especially true in asymptomatic AF patients, given that there remained a possibility that long AF persistence had already occurred before the initial hospital visit. We excluded, however, those patients in whom AF persisted for $>7$ days from the initial visit. We believe that this exclusion aided avoidance of misdiagnosis of progression into the persistent form at baseline. The present data were derived from a single hospital-based cohort in a cardiovascular hospital in an urban area of Japan. As a consequence, the present results cannot be easily extrapolated to other areas, countries, or population-based cohorts. Despite these limitations, the present data suggest the possibility that asymptomatic paroxysmal AF patients are not provided with adequate treatment, which partly contributes to disease progression and worsening of prognosis.

\section{Conclusions}

Irrespective of low-risk profile, asymptomatic paroxysmal AF patients were associated with greater AF progression to a sustained form compared to symptomatic patients. It is assumed that the paradoxical results are due to less intensive clinical management, including invasive rhythm control.

\section{Acknowledgments}

The authors have nothing to disclose. 


\section{References}

1. Go AS, Hylek EM, Phillips KA, Chang Y, Henault LE, Selby JV, et al. Prevalence of diagnosed atrial fibrillation in adults: National implications for rhythm management and stroke prevention: The AnTicoagulation and Risk Factors in Atrial Fibrillation (ATRIA) Study. JAMA 2001; 285: 2370-2375

2. Kannel WB, Abbott RD, Savage DD, McNamara PM. Epidemiologic features of chronic atrial fibrillation: The Framingham study. $N$ Engl $J$ Med 1982; 306: 1018-1022.

3. Fuster V, Rydén LE, Cannom DS, Crijns HJ, Curtis AB, Ellenbogen $\mathrm{KA}$, et al. ACC/AHA/ESC 2006 guidelines for the management of patients with atrial fibrillation - Executive summary: A report of the American College of Cardiology/American Heart Association Task Force on Practice Guidelines and the European Society of Cardiology Committee for Practice Guidelines (Writing Committee to Revise the 2001 Guidelines for the management of patients with atrial fibrillation). J Am Coll Cardiol 2006; 48: 854-906.

4. Kato T, Yamashita T, Sagara K, Fu LT. Progressive nature of paroxysmal atrial fibrillation. Circ J 2004; 68: 568-572.

5. Kerr CR, Humphries KH, Talajic M, Klein GJ, Connolly SJ, Green $\mathrm{M}$, et al. Progression to chronic atrial fibrillation after the initial diagnosis of paroxysmal atrial fibrillation: Results from the Canadian Registry of Atrial Fibrillation. Am Heart J 2005; 149: 489-496.

6. De Vos CB, Pisters R, Nieuwlaat R, Prins MH, Tieleman RG, Coelen $\mathrm{RJ}$, et al. Progression from paroxysmal to persistent atrial fibrillation. $J$ Am Coll Cardiol 2010; 55: 725-731.

7. Flaker GC, Belew K, Beckman K, Vidaillet H, Kron J, Safford R, et al. Asymptomatic atrial fibrillation: Demographic features and prognostic information from the Atrial Fibrillation Follow-up Investigation of Rhythm Management (AFFIRM) study. Am Heart J 2005; 149: 657663.

8. Senoo K, Suzuki S, Sagara K, Otsuka T, Matsuno S, Funada R, et al. Distribution of first-detected atrial fibrillation patients without structural heart diseases in symptom classifications. Circ J 2012; 76: 10201023.

9. Potpara TS, Polovina MM, Marinkovic JM, Lip GY. A comparison of clinical characteristics and long-term prognosis in asymptomatic and symptomatic patients with first-diagnosed atrial fibrillation: The Belgrade Atrial Fibrillation Study. Int J Cardiol 2013; 168: 4744-
4749

10. Suzuki S, Yamashita T, Otsuka T, Sagara K, Uejima T, Oikawa Y, et al. Prevalence and prognosis of patients with atrial fibrillation in Japan: A prospective cohort of Shinken Database 2004. Circ J 2008; 72: 914-920.

11. Suzuki S, Yamashita T, Otsuka T, Sagara K, Uejima T, Oikawa Y, et al. Recent mortality of Japanese patients with atrial fibrillation in an urban city of Tokyo. J Cardiol 2011; 58: 116-123.

12. Suzuki S, Yamashita T, Ohtsuka T, Sagara K, Uejima T, Oikawa Y, et al. Body size and atrial fibrillation in Japanese outpatients. Circ J 2010; 74: $66-70$.

13. Suzuki S, Otsuka T, Sagara K, Matsuno S, Funada R, Uejima T, et al. Dabigatran in clinical practice for atrial fibrillation with special reference to activated partial thromboplastin time. Circ J 2012; 76: $755-$ 757.

14. Suzuki S, Sagara K, Otsuka T, Matsuno S, Funada R, Uejima T, et al. Gender-specific relationship between serum uric acid level and atrial fibrillation prevalence. Circ J 2012; 76: 607-611.

15. Gage BF, Waterman AD, Shannon W, Boechler M, Rich MW, Radford MJ. Validation of clinical classification schemes for predicting stroke: Results from the National Registry of Atrial Fibrillation. JAMA 2001; 285: $2864-2870$.

16. Lip GY, Nieuwlaat R, Pisters R, Lane DA, Crijns HJ. Refining clinical risk stratification for predicting stroke and thromboembolism in atrial fibrillation using a novel risk factor-based approach: The Euro Heart Survey on Atrial Fibrillation. Chest 2010; 137: 263-272.

17. Flaker GC, Belew K, Beckman K, Vidaillet H, Kron J, Safford R, et al. Asymptomatic atrial fibrillation: Demographic features and prognostic information from the Atrial Fibrillation Follow-up Investigation of Rhythm Management (AFFIRM) study. Am Heart J 2005; 149: 657-663.

18. Wyse DG, Waldo AL, DiMarco JP, Domanski MJ, Rosenberg Y, Schron EB, et al. Atrial Fibrillation Follow-up Investigation of Rhythm Management (AFFIRM) Investigators. A comparison of rate control and rhythm control in patients with atrial fibrillation. $N$ Engl J Med 2002; 347: 1825-1833.

19. Eckstein J, Verheule S, de Groot NM, Allessie M, Schotten U. Mechanisms of perpetuation of atrial fibrillation in chronically dilated atria. Prog Biophys Mol Biol 2008; 97: 435-451. 